\title{
Protección contra herbivoría en reforestación de terreno árido degradado, con palo verde (Parkinsonia microphylla Torr.)
}

\section{Protection against herbivory in reforestation of degraded arid field, with Palo Verde (Parkinsonia microphylla Torr.)}

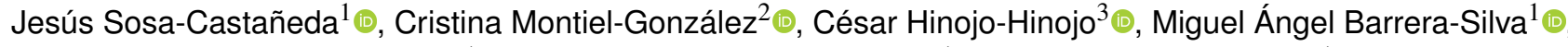

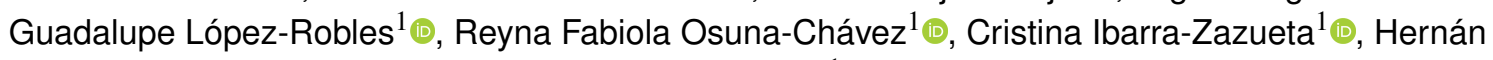 \\ Celaya-Michel ${ }^{1 *}$ (i) \\ ${ }^{1}$ Departamento de Agricultura y Ganadería, Universidad de Sonora, Km 21 Carretera Bahía de Kino, CP. 83000. Hermosillo, Sonora, \\ México. \\ ${ }^{2}$ Escuela Nacional de Estudios Superiores. Morelia, Antigua Carretera a Pátzcuaro No. 8701, CP. 58190. Morelia, Michoacán, México. \\ ${ }^{3}$ Department of Earth System Science, University of California, Irvine. CA 92697, USA. \\ *Autor de correspondencia: hernan.celaya@unison.mx
}

Nota científica recibida: 13 de noviembre de 2018 aceptada: 06 de agosto 2019

RESUMEN. Rehabilitar terrenos degradados áridos, demanda superar limitaciones bióticas y abióticas. El objetivo del estudio fue conocer si refugios protectores de bajo costo, mejoran la supervivencia y el crecimiento de plantas de palo verde (Parkinsonia microphylla), durante el primer año de reforestación en sitios degradados del Desierto Sonorense. Se construyeron refugios protectores reutilizando desechos de poda y envases plásticos. Durante un año, 264 plantas de palo verde fueron sometidas a tratamientos diferenciales de protección contra herbívoros (sin protección, y con protección por refugios de ramas, PET o ambos), y se monitoreó su supervivencia y altura. La supervivencia de plantas con el uso de refugios protectores fue mayor que la de las plantas sin protección (34.9-56.7 vs. $\left.1.8 \%, \chi^{2}=63.5 ; p<0.0001\right)$; también las alturas de las plantas protegidas fueron mayores. El uso de refugios es una alternativa para la reforestación con palo verde en terrenos degradados del desierto Sonorense.

Palabras clave: Biodiversidad, costa de Hermosillo, desertificación, Desierto Sonorense.

ABSTRACT. Rehabilitate degraded arid lands, demand to overcome biotic and abiotic limitations. The objective of the study was to know if low-cost protective shelters, improve the survival and growth of palo verde (Parkinsonia microphylla), during the first year of reforestation in degraded sites of the Sonoran Desert. Protective shelters were built reusing pruning waste and plastic containers. During a year, 264 plants of palo verde were subjected to differential treatments of protection against herbivores (without protection, and with protection by stick, PET or both), and their survival and height were monitored. The survival of plants with the use of protective shelters was greater than that of plants without protection (34.9-56.7 vs. $1.8 \%, \chi^{2}$ $=63.5, p<0.0001)$; likewise, the heights of the protected plants were higher. The use of shelters is an effective, alternative for the reforestation with palo verde in degraded lands within the Sonoran Desert.

Key words: Biodiversity, desertification, Hermosillo coast, Sonoran Desert.

\section{INTRODUCCIÓN}

La degradación de terrenos, es un problema que preocupa a la humanidad desde hace varias décadas (Reynolds y Stafford-Smith 2002, Blaikie y Brookfield 2015). Esta degradación afecta de forma negativa la seguridad alimentaria y el bienestar hu- mano (Pagiola 1999, Hassan et al. 2005). Por esta razón, hoy en día se ha vuelto necesario desarrollar métodos que ayuden a restaurar los terrenos degradados. Actualmente se desconoce la situación de pérdida de suelos en gran parte del mundo y en México (Turner et al. 2016). Sin embargo, existen indicadores que sugieren que las 
zonas áridas y semiáridas son las más vulnerables a la degradación de terrenos en comparación con otros ecosistemas (Millennium Ecosystem Assessment 2005, Blaikie y Brookfield 2015). Un problema sumamente importante que ha generado esta degradación de terrenos es la desertificación de las zonas áridas y semiáridas del mundo. Se ha proyectado que para el 2100 , las zonas áridas y semiáridas aumentarán del 11 al $23 \%$ del territorio global (Huang et al. 2016). Así mismo, aproximadamente, el $70 \%$ de las zonas secas productivas están actualmente amenazadas por diversas formas de desertificación y degradación (Huang et al. 2016).

La restauración natural en ecosistemas de zonas áridas degradadas puede ser muy lenta (Abella 2010). Por ello, la reforestación de zonas áridas es una alternativa obligada (Blaikie y Brookfield 2015), que representa retos, como: a) condiciones abióticas desfavorables para el desarrollo de las plantas la mayor parte del año, como la baja disponibilidad de agua (Whitford 2002) y temperaturas elevadas, tanto ambientales y de la superficie del suelo (Balba 2018); y b) las condiciones bióticas estresantes como los altos niveles de herbivoría que afectan el desarrollo de la vegetación (Ward 2009).

En las regiones áridas de México la reforestación se realiza por medio de programas de gobierno y dependencias oficiales (CONAFOR 2010, Dorado y Arias 2018). Sin embargo, los esfuerzos de reforestación se ven limitados por la poca eficiencia con que se hace frente a las condiciones abióticas y de herbivoría típicas de las zonas áridas (Bowers et al. 2004, Moreno et al. 2017). Por ello, la protección contra herbívoros y la mitigación de las condiciones desfavorables podrían ser provistas artificialmente a través de refugios protectores de plantas (Bainbridge 1994, Callaway 1995). Los cuales se pueden adquirir comercialmente o elaborarse con diversos materiales, aunque su uso en áreas extensivas aumenta los costos de reforestación (CONAFOR 2010).

El trabajo se enfocó en el estudio del palo verde (Parkinsonia microphylla Torr.) como una planta con potencial para reforestación de terrenos degradados del desierto Sonorense. Por su amplia distribución, resistencia a la sequía y capacidad como planta nodriza el palo verde, es una especie clave en los ecosistemas del Desierto Sonorense (Turner et al. 1995). Además es una especie muy palatable para los herbívoros lo cual ocasiona bajas tasas de sobrevivencia de plantas durante los primeros años de vida (Bowers et al. 2004, Moreno et al. 2017). Por lo anterior el objetivo del estudio fue conocer si los refugios protectores de bajo costo, mejoran la supervivencia y el crecimiento de plantas de palo verde, durante el primer año post trasplante en sitios degradados del Desierto Sonorense.

\section{MATERIALES Y MÉTODOS}

\section{Área de estudio}

El área de estudio se ubica en el Desierto Sonorense, en el municipio de Hermosillo, Sonora, en el campo experimental del Departamento de Agricultura y Ganadería de la Universidad de Sonora. Tiene 244 hectáreas de extensión, ubicado en las coordenadas $29^{\circ} 01^{\prime} 13^{\prime \prime}$ LN y $111^{\circ} 08^{\prime} 05^{\prime \prime}$ LO, con altitud de $191 \mathrm{msnm}$. Originalmente, la vegetación predominante era matorral, pero ha sido transformada a terrenos agrícolas y áreas desmontadas donde se sembró zacate buffel (Pennisetum ciliare (L.) Link) para pastoreo de ganado bovino. El clima es muy seco y muy cálido, con temperatura media anual de $25.2{ }^{\circ} \mathrm{C}$, las temperaturas máximas se registran en el mes de junio y las mínimas en febrero. Las lluvias principalmente se presentan durante el monzón de verano entre los meses de julio y agosto (INEGI 2014), con precipitación media anual de $378 \mathrm{~mm}$. El tipo de suelo del sitio es arenoso franco, con baja disponibilidad de nutrientes (WRB 2015).

Se evaluó el suelo desnudo, con la metodología de la línea de Canfield (Canfield 1941), en un área de 20 ha, con pendiente del 3\%, excluida recientemente del pastoreo de bovinos. El suelo desnudo domina el paisaje en un $70 \%$. Se observó en el área de estudio, la presencia de herbívoros pequeños como: liebres (Lepus californicus) y juansitos (Xerospermophilus tereticaudus), con menor frecuencia conejos, así como huellas de venado bura (Odocoileus hemionus). 
Colecta de semillas y establecimiento de plantas

Para la colecta de semilla de palo verde, se obtuvo el permiso de colecta científica de la SEMARNAT. Las semillas de palo verde se sometieron a tratamientos de germinación en vivero en agosto de 2016. No se utilizó escarificación en las semillas. Se sembró una semilla por maceta de $1.5 \mathrm{~L}$ de volumen con suelo del terreno donde se trasplantarían, se regaron cada cuatro días con $0.25 \mathrm{~L}$ de agua por maceta, y permanecieron bajo malla sombra durante 2 meses. Posteriormente, en octubre del 2016, un total de 300 plántulas jóvenes (de 60 días de edad), se trasplantaron al agostadero degradado.

Cuando las plantas tenían una altura entre 37.88 y $46.07 \mathrm{~cm}$ (Tabla 1), se trasplantaron a una profundidad de $20 \mathrm{~cm}$, con separación de $10 \mathrm{~m}$ entre planta. Del número inicial de individuos trasplantados, en la primera semana, se establecieron con éxito 264 , lo que indica una sobrevivencia del $88 \%$.

\section{Diseño experimental}

Se utilizó un diseño factorial de cuatro niveles que corresponden a tratamientos de protección a herbivoría (sin protección, palos, PET y palos-PET). Los niveles del diseño experimental se distribuyeron de la siguiente forma, las 264 plantas establecidas, se dividieron en dos grupos, los cuales incluyen 150 plantas protegidas contra la herbivoría y 114 plantas sin protección a herbivoría. Así mismo, la protección consistió en tres tratamientos: 1) Tratamiento Palos, a 43 plantas, por medio de la colocación varas secas o palos de árboles de la región que se enterraron alrededor de la planta a un diámetro de $20 \mathrm{~cm}$, y altura de $30 \mathrm{~cm}$ o más; 2) Tratamiento PET, a 77 plantas, a las que se les colocó un recipiente plástico de tereftalato de polietileno, procedente de recipientes reutilizados de refresco de $3 \mathrm{~L}$, con dimensiones de 30 $\mathrm{cm}$ de alto y diámetro de $12 \mathrm{~cm}$, que se enterraron a profundidad de $5 \mathrm{~cm}$; y 3) Tratamiento palo-PET, realizado a 30 plantas, en este tratamiento se colocó el recipiente plástico PET y además las varas secas o palos circundando a la planta. Se aplicó un riego de $3 \mathrm{~L}$ de agua a cada planta al trasplante, y dos riegos de auxilio de $2 \mathrm{~L}$ de agua, cada 15 días posteriores al trasplante.
Las variables de respuesta evaluadas fueron: supervivencia y altura, las cuales se evaluaron al trasplante, a los 10, 20, 30, 90, 150, 230, 270 y 365 días posteriores. Así mismo, la supervivencia se evaluó contabilizando las plantas vivas encontradas, con respecto a las 264 plantas que inicialmente se establecieron. Para determinar la altura se realizaron mediciones en campo con una cinta métrica, con la cual se comparó la diferencia entre la altura inicial de las plantas con respecto a la misma al término de cada periodo de muestreo. Adicionalmente, se determinó si los tratamientos de protección causaban un microambiente más favorable o no, para las plantas durante el verano, periodo en que ocurren las temperaturas más altas del año (Whitford 2002). Para la evaluación, se determinó la temperatura del suelo con un datalogger (Micrologger 21X, Campbell scientific Inc.) con dos termopares tipo T. La medición se llevó a cabo en una sola ocasión, a las 15 horas, en agosto, en el suelo de 20 plantas al azar por tratamiento.

\section{Análisis estadísticos}

Los análisis estadísticos se realizaron con el programa JMP versión 10 (SAS 2000). Con los datos de supervivencia se realizó una tabla de contingencia con la prueba de ji cuadrada $\left(\chi^{2}\right)$ de acuerdo con Infante y Zarate-de Lara (2000). El resto de la información correspondiente a la altura de las plantas, se contrastó mediante análisis de varianza. La prueba a posteriori utilizada fue Tukey. En todos los casos se estableció una significancia estadística menor o igual al $5 \%(p \leq 0.05)$.

\section{RESULTADOS Y DISCUSIÓN}

A partir de los 20 días de establecimiento de las plántulas de palo verde, se encontraron diferencias significativas entre los tratamientos de protección para la variable supervivencia $(p=0.0002,100$ vs $91.2 \%$, Figura 1). Se observó mayor supervivencia en las plantas con protección a herbivoría, con respecto a las plantas sin protección. Para las plantas sin protección, se observó un rápido descenso en la tasa de supervivencia. A los 90 días después del trasplante, 
Tabla 1. Medias y desviación estándar (DE) de las alturas $(\mathrm{cm})$ de palo verde (Parkinsonia microphylla Torr.) desde el trasplante hasta el año de establecimiento. En cada etapa, los valores seguidos verticalmente por diferentes letras indican que las medias son significativamente diferentes.

\begin{tabular}{|c|c|c|c|c|c|c|c|c|c|}
\hline Etapa & Tratamiento & Número & Media & DE & & & & $\mathrm{F}$ & Valor $p$ \\
\hline \multirow{4}{*}{ Trasplante } & Palos-PET & 30 & 46.07 & 11.73 & $a$ & & & 2.6342 & 0.0503 \\
\hline & Palos & 43 & 43.47 & 14.92 & a & & & & \\
\hline & PET & 77 & 37.88 & 12.49 & a & & & & \\
\hline & Sin protección & 114 & 41.36 & 17.19 & a & & & & \\
\hline \multirow[t]{4}{*}{10 días } & Palos-PET & 30 & 45.80 & 12.01 & a & & & 8.3185 & $<0.0001$ \\
\hline & Palos & 43 & 40.11 & 18.56 & a & $\mathrm{b}$ & & & \\
\hline & PET & 77 & 33.19 & 14.18 & & b & c & & \\
\hline & Sin protección & 114 & 28.91 & 22.57 & & & c & & \\
\hline \multirow[t]{4}{*}{20 días } & Palos-PET & 30 & 41.52 & 11.43 & a & & & 10.9551 & $<0.0001$ \\
\hline & Palos & 43 & 37.37 & 20.56 & a & b & & & \\
\hline & PET & 77 & 29.74 & 15.28 & & b & c & & \\
\hline & Sin protección & 104 & 22.18 & 23.38 & & & c & & \\
\hline \multirow[t]{4}{*}{30 días } & Palos-PET & 30 & 40.35 & 10.26 & a & & & 52.8729 & $<0.0001$ \\
\hline & Palos & 38 & 23.57 & 16.75 & & b & & & \\
\hline & PET & 75 & 23.88 & 10.65 & & b & & & \\
\hline & Sin protección & 79 & 7.64 & 13.41 & & & c & & \\
\hline \multirow[t]{4}{*}{90 días } & Palos-PET & 29 & 40.97 & 17.56 & a & & & 21.619 & $<0.0001$ \\
\hline & Palos & 35 & 22.94 & 15.68 & & b & & & \\
\hline & PET & 74 & 22.04 & 10.81 & & b & & & \\
\hline & Sin protección & 34 & 12.65 & 15.77 & & & c & & \\
\hline \multirow[t]{4}{*}{150 días } & Palos-PET & 28 & 39.36 & 19.95 & a & & & 17.5924 & $<0.0001$ \\
\hline & Palos & 33 & 24.76 & 15.20 & & b & & & \\
\hline & PET & 67 & 19.19 & 9.53 & & b & c & & \\
\hline & Sin protección & 23 & 13.49 & 15.77 & & & c & & \\
\hline \multirow[t]{4}{*}{230 días } & Palos-PET & 26 & 42.27 & 21.38 & a & & & 13.6253 & $<0.0001$ \\
\hline & Palos & 28 & 21.43 & 17.19 & & b & & & \\
\hline & PET & 49 & 18.92 & 10.72 & & $b$ & & & \\
\hline & Sin protección & 13 & 18.21 & 17.98 & & $\mathrm{~b}$ & & & \\
\hline \multirow[t]{4}{*}{270 días } & Palos-PET & 21 & 39.29 & 9.10 & a & & & 11.5018 & $<0.0001$ \\
\hline & Palos & 19 & 25.68 & 17.70 & & b & & & \\
\hline & PET & 38 & 19.97 & 11.63 & & b & & & \\
\hline & Sin protección & 4 & 12.75 & 16.21 & & b & & & \\
\hline \multirow[t]{4}{*}{365 días } & Palos-PET & 17 & 42.29 & 5.79 & a & & & 9.3302 & $<0.0001$ \\
\hline & Palos & 15 & 31.00 & 19.49 & a & b & & & \\
\hline & PET & 32 & 21.75 & 11.95 & & b & & & \\
\hline & Sin protección & 2 & 21.50 & 21.92 & a & b & & & \\
\hline
\end{tabular}

la supervivencia fue del $29.8 \%$ de las plantas sin protección, mientras que, para la misma fecha, las plantas con protección tenían $92 \%$ de supervivencia ( $p$ $<0.0001$ ); ambos tratamientos presentaron mortalidad de plantas con el paso de los meses. Pero la protección favoreció la supervivencia $(p<0.0001)$, ya que después de 12 meses se observó una supervivencia del $43 \%$ de las plantas con protección y $1.8 \%$ de las plantas sin protección. Al respecto Bowers et al. (2004) indican que mejorar la supervivencia de plantas para reforestación durante su primer año de vida es fundamental, ya que en este periodo ocurren las mayores tasas de mortalidad. Al respecto Hackworth et al. (2018) encontraron, mayor supervivencia con protección en dos de tres especies arbóreas.
La altura de las plantas, indica que con el paso de los días la altura disminuyó con diferencias significativas en la altura entre tratamientos (Tabla 1). A partir de los 10 días, la mayor diferencia de altura se obtuvo en el tratamiento de las plantas protegidas con palo-PET, que solo vieron disminuida su altura inicial en $0.5 \%$, mientras que las plantas sin protección tuvieron una reducción de altura del $30 \%$, apreciándose en campo daños de cortes por herbívoros. Se observó que a los 30 días, las plantas con protección eran de 3 a 5 veces más altas, que las plantas del tratamiento sin protección. De los 90 días en adelante, el porcentaje de plantas vivas sin protección fue del 1.8\%, quedando de 114 plantas sólo dos plantas vivas al año, por lo que no se pudo realizar un análi- 


\section{$\%$ de Supervivencia}

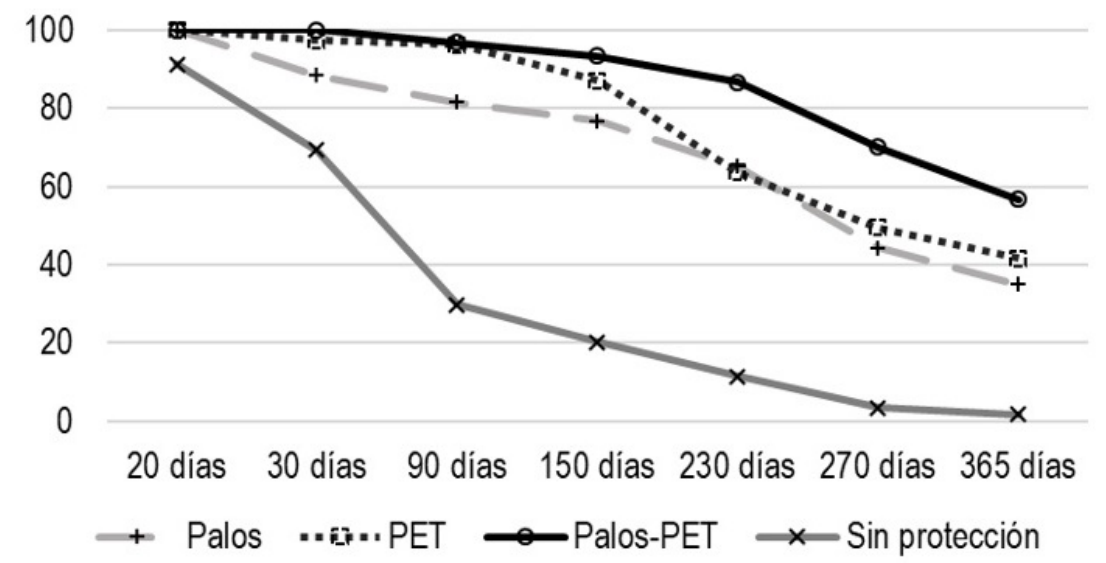

Figura 1. Supervivencia de palo verde (Parkinsonia microphylla Torr.) con diferente protección contra herbívoros. Por fecha de muestreo $\left(\chi^{2}: 13.6,35.1,113.2,123.4,91.1,77.1\right.$ y 63.5 ; valor $p$ : $0.0034,<0.0001,<0.0001,<0.0001,<0.0001,<0.0001,<0.0001$, respectivamente).

sis estadístico de la altura (Tabla 1). Al respecto, se sabe que el consumo de las plantas por herbivoría es la principal causa de mortalidad en etapas tempranas en palo verde y en otras especies perennes del desierto Sonorense (Boyd y Brum 1983, McAuliffe 1986, Bowers et al. 2004). Lo anterior, se constató en el presente estudio, ya que la herbivoría ocasionó la disminución en la altura de las plantas. Estudios recientes con maya protectora de $46 \mathrm{~cm}$ de alto encontraron, diferencia numérica, sin ser estadísticamente significativa. En el presente estudio se observaron herbívoros de fauna menor, como liebres, conejos y juansitos, consumiendo las plantas evaluadas, otros estudios, han encontrado que los cérvidos tuvieron más importancia en la herbivoría (Hackworth et al. 2018). Aunque también se reportaron resultados con especies invasoras de conejos, que afectaron la sobrevivencia y crecimiento de plantas trasplantadas (Forsyth et al. 2015). Además de estudios en coníferas que mostraron mayor reclutamiento de plantas en las exclusiones a todos los herbívoros, en particular los conejos (Zimmer et al. 2017).

Los tratamientos de protección tuvieron suelos con temperaturas más bajas que los suelos con plantas sin protección (Figura $2, p<0.0001$ ), en los momentos que se espera mayor temperatura del (Whitford 2002). Los diferentes tratamientos de protección disminuyeron la temperatura del suelo entre 11 y 17.7 ${ }^{\circ} \mathrm{C}$ en comparación con las plantas que no tenían protección y que alcanzaron temperaturas superiores a los $60{ }^{\circ} \mathrm{C}$. Además de favorecer la supervivencia de las plantas, los tratamientos de protección también generaron un microambiente favorable para el desarrollo de las plantas (Figura 2). Este resultado es consistente con otros en los que se reporta que la temperatura influye en las plantas de pequeña altura (Ehleringer 1985, Balba 2018), como el caso de los árboles durante su primer año de vida, utilizados en el presente estudio. Al mejorar el microambiente al que están expuestas las plantas se reducen los niveles de estrés ambiental, lo que permite su establecimiento. En un gradiente de aridez en Mongolia, se encontró que los arbustos proveían un microambiente y protección contra herbivoría, que favoreció el establecimiento de las plantas (Xie et al. 2017).

Los resultados sugieren que el uso de refugios protectores elaborados a partir de la reutilización de desechos es una metodología efectiva para favorecer la supervivencia de plantas nativas en su primer año de vida, en sitios áridos degradados. El porcentaje de supervivencia obtenido por la protección de estos refugios fue del 34.9 al $56.7 \%$, lo cual es comparable con el que ha sido obtenido en reforestaciones 


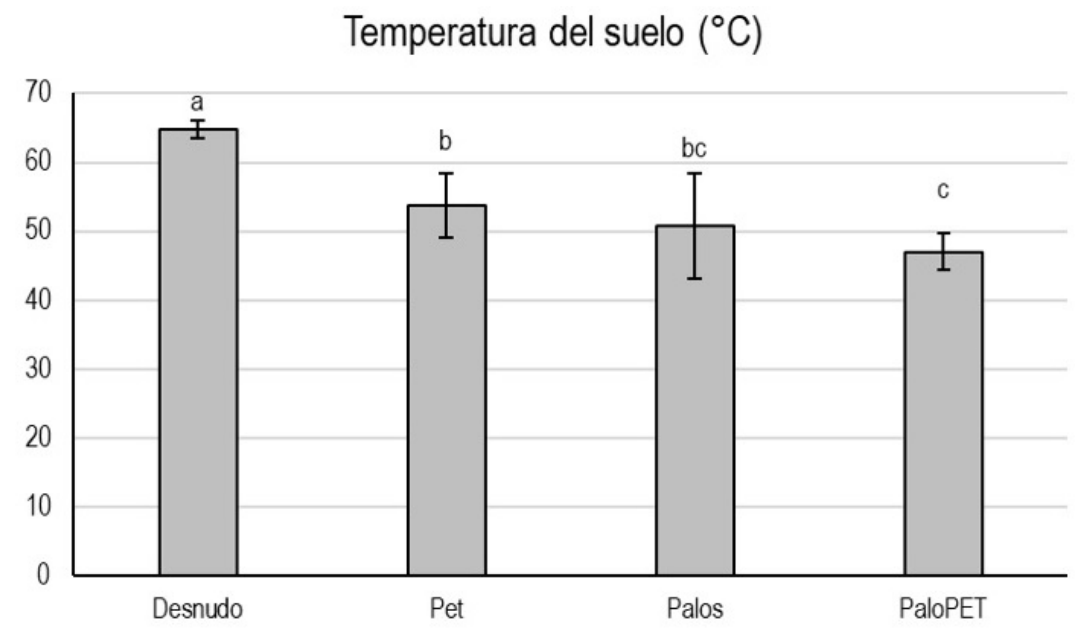

Figura 2. Efecto de los tratamientos de protección sobre la temperatura del suelo al mediodía, durante el verano. Diferentes letras indican que las medias entre tratamientos son significativamente diferentes $(p<0.05)$.

exitosas con el uso de plantas nodrizas (Rey et al. 2009). El uso de refugios protectores en la reforestación de ecosistemas áridos es muy variable. Por un lado, refugios elaborados con PVC tienen una supervivencia de alrededor del $20 \%$, mientras que los refugios comerciales tienen supervivencias de entre el 40 y $80 \%$ (Bainbridge 1994). También se han reportado supervivencias de entre 60 y $95 \%$ para Quercus coccifera, y supervivencias de entre 0 y $24 \%$ para otras especies nativas de sitios semiáridos de España (Bellot et al. 2002, Padilla et al. 2011). Por otra parte, las prácticas tradicionales de reforestación en las que se siembran las plantas sin ninguna protección por lo general resultan en sobrevivencias menores al $20 \%$ e incluso nula supervivencia (Rey et al. 2009, CONAFOR 2010). Por lo que los tratamientos propuestos pueden ser una alternativa económica viable para la reforestación de sitios áridos degradados, y al mismo tiempo aprovechar materiales que de otra manera se desperdiciarían o que contribuyen a la generación de residuos sólidos de difícil degradación. Por lo que se debe continuar con las investigaciones que permitan evaluar la efectividad de diferentes tipos de protecciones contra las condiciones abióticas y bióticas, en otras especies con fines de reforestación. Pero es poco el conocimiento sobre los efectos de los diferentes tipos de refugios y/o protecciones, sobre el ambiente que experimenta la planta; además de que se desconoce el efecto del microambiente en el desempeño fisiológico de la planta (Williams y Balling 1996).

La herbivoría influencia el reclutamiento de plantas nativas forrajeras trasplantadas en terrenos degradados del Desierto Sonorense. Los tratamientos que no utilizaron alguna protección para las plantas tuvieron una supervivencia nula al paso de un año. Es factible incrementar la supervivencia de plantas forrajeras nativas trasplantadas en terrenos degradados, mediante la protección contra herbívoros de fauna menor, como liebres, conejos y juansitos, con el uso de refugios hechos a partir de materiales como ramas de poda y envases plásticos reutilizados.

\section{AGRADECIMIENTOS}

A la Universidad de Sonora, División de Investigación y Posgrado, División de Ciencias Biológicas y de la Salud, Departamento de Agricultura y Ganadería, por el apoyo de los proyectos USO313002719 y USO313003110. 


\section{LITERATURA CITADA}

Abella SR (2010) Disturbance and plant succession in the Mojave and Sonoran Deserts of the American Southwest. International Journal of Environmental Research and Public Health 7: 1248-1284.

Bainbridge DA (1994) Tree shelters improve establishment on dry sites. Tree Plant Notes 45: 13-16.

Balba A (2018) Management of problem soils in arid ecosystems. Boca Raton, CRC Press. Boca Raton FL, USA. 250p.

Blaikie P, Brookfield H (2015) Land degradation and society. Routledge. New York, USA. 296p.

Bellot J, Ortiz De Urbina JM, Bonet A, Sánchez JR (2002) The effects of treeshelters on the growth of Quercus coccifera L. seedlings in a semiarid environment. Forestry 75: 89-106.

Bowers JE, Turner RM, Burgess TL (2004) Temporal and spatial patterns in emergence and early survival of perennial plants in the Sonoran Desert. Plant Ecology 172: 107-119.

Boyd RS, Brum GD (1983) Postdispersal reproductive biology of a Mojave Desert population of Larrea tridentata (Zigophyllaceae). American Midland Naturalist 110: 25-36.

Callaway RM (1995) Positive interactions among plants. The Botanical Review 61: 306-349.

Canfield RH (1941) Application of the line interception method in sampling range vegetation. Journal of Forestry 39: 388-394.

CONAFOR (2010) Prácticas de reforestación: Manual Básico. Comisión Nacional Forestal, Zapopan, Jalisco, México. 62p.

Dorado O, Arias D (2018) Reforestar o restaurar para la recuperación ambiental. Inventio, Génesis de la Cultura Universitaria en Morelos 3: 34-38.

Ehleringer J (1985) Annuals and perennials of warm deserts. In: Chabot BF, Mooney HA (eds) Physiological ecology of North American plant communities. Chapman and Hall. London, UK. pp: 162-180.

Forsyth DM, Scroggie MP, Arthur AD, Lindeman M, Ramsey DSL, McPhee SR, et al. (2015) Density-dependent effects of a widespread invasive herbivore on tree survival and biomass during reforestation. Ecosphere 6 : Article 71. Doi: 10.1890/ES14-00453.1.

Hackworth Z, Lhotka J, Cox J, Barton, C, Springer M (2018) First-year vitality of reforestation plantings in response to herbivore exclusion on reclaimed appalachian surface-mined land. Forests 9: 222. Doi: 10.3390/f9040222.

Hassan R, Scholes R, Ash N (2005) Ecosystems and human well-being: Current State and Trends, Volume 1. Island Press, Washington, DC, USA. 917p.

Huang J, Yu H, Guan X, Wang G, Guo R (2016) Accelerated dryland expansion under climate change. Nature Clim Change 6: 166-171.

INEGI (2014) Anuario estadístico y geográfico de Sonora 2014. Instituto Nacional de Estadística y Geografía. México. 611p.

Infante GS, Zarate-de Lara GP (2000) Métodos estadísticos: un enfoque interdisciplinario. Sexta reimpresión. Editorial Trillas. México. 643p.

McAuliffe JR (1986) Herbivore-limited establishment of a Sonoran Desert tree, Cerciduium Microphyllum. Ecology 67: $276-280$. 
Millennium Ecosystem Assessment (2005) Bromus tectorum ecosystems and human Well-Being: Desertification Synthesis. World Resources Institute, Washington, USA. 155p.

Moreno M, de-Bashan LE, Hernandez JP, Lopez BR, Bashan Y (2017) Success of long-term restoration of degraded arid land using native trees planted 11 years earlier. Plant and Soil 421: 83-92.

Padilla FM, Miranda J de D, Ortega R, Hervás M, Sánchez J, Pugnaire FI (2011) Does shelter enhance early seedling survival in dry environments? A test with eight Mediterranean species. Applied Vegetation Science 14: 31-39.

Pagiola S (1999) The global environmental benefits of land degradation control on agricultural land: Global overlays program. The World Bank. Washington, USA. 46p.

Rey PJ, Siles G, Alcántara JM (2009) Community-level restoration profiles in Mediterranean vegetation: nursebased vs. traditional reforestation. Journal of Applied Ecology 46: 937-945.

Reynolds JF, Stafford-Smith DM (2002) Do humans cause deserts? In: Reynolds JF, Stafford-Smith DM (Eds.). Global desertification: Do humans cause deserts? Dahlem Workshop Report 88. Dahlem University Press. Berlin, Germany. pp: 1-21

SAS (2000) SAS statistical software. Version 5. SAS Institute. Cary, NC, USA. 254p.

Turner RM, Bowers JE, Burgess TL (1995) Sonoran desert plants: An ecological atlas. The University of Arizona Press, Arizona, USA. 511p.

Turner KG, Anderson S, Gonzales-Chang M, Costanza R, Courville S, Dalgaard T, et al. (2016) A review of methods, data, and models to assess changes in the value of ecosystem services from land degradation and restoration. Ecological Modelling 319: 190-207.

Ward D (2009) The biology of deserts. Oxford University Press. New York, NY, USA. 370p.

Whitford WG (2002) Ecology of Desert Ecosystems. Elsevier Science Ltd. London, UK. San Diego, CA, USA. $343 p$.

Williams MA, Balling Jr RC (1996) Interactions of desertification and climate. Edward Arnold, Hodder Headline, PLC. London, UK. 270p.

WRB (2015) World reference base for soil resources 2014, update 2015. World Soil Resources Reports No. 106. FAO. Rome, Italy. 192p.

Xie LN, Guo HY, Liu Z, Gabler CA, Chen WZ, Gu S, Ma, CC (2017) Shrubs facilitate recruitment of Caragana stenophylla Pojark: microhabitat amelioration and protection against herbivory. Annals of Forest Science 74: 70. Doi: 10.1007/s13595-017-0668-4.

Zimmer HC, Florentine SK, Enke R, Westbrooke M (2017) Rainfall and grazing: not the only barriers to arid-zone conifer recruitment. Australian Journal of Botany 65: 109-119. 